Published in final edited form as:

Curr Opin Virol. 2020 October ; 44: 16-25. doi:10.1016/j.coviro.2020.05.004.

\title{
Reverse genetics approaches for the development of Bunyavirus vaccines
}

\author{
Breanna Tercero ${ }^{1}$, Shinji Makino ${ }^{*}, 1,2,3,4,5$ \\ ${ }^{1}$ Departments of Microbiology and Immunology and, the University of Texas Medical Branch, \\ Galveston, Texas 77555-1019 \\ ${ }^{2}$ Institute of Human Infection and Immunity and, the University of Texas Medical Branch, \\ Galveston, Texas 77555-1019 \\ ${ }^{3}$ Center for Biodefense and Emerging Infectious Diseases and, the University of Texas Medical \\ Branch, Galveston, Texas 77555-1019 \\ ${ }^{4}$ UTMB Center for Tropical Diseases and, the University of Texas Medical Branch, Galveston, \\ Texas 77555-1019
}

${ }^{5}$ The Sealy Institute for Vaccine Sciences and, the University of Texas Medical Branch, Galveston, Texas 77555-1019

\section{Abstract}

The Bunyavirales order is the largest group of RNA viruses, which includes important human and animal pathogens, that cause serious diseases. Licensed vaccines are often not available for many of these pathogens. The establishment of bunyavirus reverse genetics systems has facilitated the generation of recombinant infectious viruses, which have been employed as powerful tools for understanding bunyavirus biology and identifying important virulence factors. Technological advances in this area have enabled the development of novel strategies, including codondeoptimization, viral genome rearrangement and single-cycle replicable viruses, for the generation of live-attenuated vaccine candidates. In this review, we have summarized the current knowledge of the bunyavirus reverse genetics approaches for the generation of live-attenuated vaccine candidates and their evaluation in animal models.

\section{Graphical Abstract}

*Corresponding author. Mailing address: Department of Microbiology and Immunology, The University of Texas Medical Branch, 4.142E Medical Research Building, 301 University Boulevard, Galveston, Texas 77555-1019. Phone: (409) 772-2323. Fax: (409) 772-5065.shmakino@utmb.edu.

Publisher's Disclaimer: This is a PDF file of an unedited manuscript that has been accepted for publication. As a service to our customers we are providing this early version of the manuscript. The manuscript will undergo copyediting, typesetting, and review of the resulting proof before it is published in its final form. Please note that during the production process errors may be discovered which could affect the content, and all legal disclaimers that apply to the journal pertain.

Declaration of Competing Interest

The author(s) declare no competing interests. 


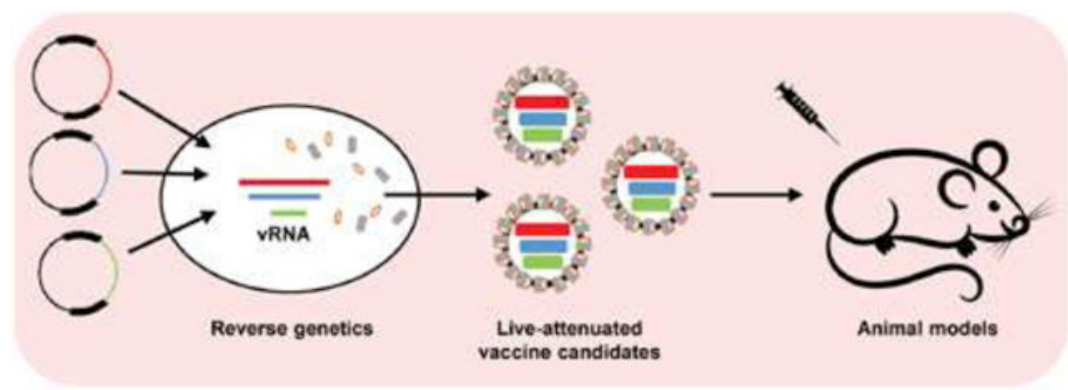

\section{Keywords}

Bunyaviruses; Reverse genetics systems; Vaccines

\section{Introduction}

Among the 12 families in the Bunyavirales order [1], Arenaviridae, Hantaviridae,

Nairoviridae, Peribunyaviridae and Phenuiviridae have been known to contain important pathogens that cause severe diseases in humans and animals. Transmission of pathogenic bunyaviruses to humans can occur by being bitten by infected arthropod vectors, close contact with infected tissue or fluids, or exposure to the excretion of infected rodents [2-7]. Several arenaviruses, including Lassa virus (LASV), Machupo virus (MACV), and Junin virus (JUNV), cause severe hemorrhagic fever diseases [2,8]. Viruses in the Hantaviridae family, such as Andes virus (ANDV), cause hantavirus cardiopulmonary syndrome and a severe respiratory disease with high mortality rates [5,7]. Crimean-Congo hemorrhagic fever virus (CCHFV) of Nairoviridae, which is widely distributed across Asia, Africa, the Middle east and Europe, can cause severe hemorrhagic fever with high fatality rates [3,7,9]. Members of the Peribunyaviridae family, including La Crosse virus (LACV) and Oropouche virus (OROV), have been linked to encephalitis leading to significant neurological sequelae [6,7]. Rift Valley fever phlebovirus (RVFV), found within the family Phenuiviridae, can cause severe symptoms including ocular damage, hemorrhagic fever, and late-onset encephalitis [4,7]. Severe fever with thrombocytopenia syndrome virus (SFTSV) of Phenuiviridae causes thrombocytopenia, leukocytopenia, and hemorrhagic fever [7]. Bunyaviruses also pose a serious threat to agriculture due to severe disease in ruminants. Schmallenberg virus (SBV) of the Peribunyaviridae family causes abortions and malformed newborns in cattle [10]. RVFV infection in young livestock shows high mortality rates and can lead to "abortion storms" in pregnant animals, causing a significant negative impact on the economy [4].

One of the best strategies to control bunyavirus infections is through the generation of vaccines by using reverse genetics systems. The objective of this review is to highlight and summarize the current knowledge of the reverse genetic approaches for vaccine development against important human and animal pathogens in the Bunyavirales order. 


\section{Bunyavirus genome organization}

Bunyaviruses are enveloped viruses that consist of a segmented, negative sense, singlestranded RNA genome. Bunyaviruses, except for Arenaviridae, have a tri-segmented RNA genome comprised of L, M, and S segments. Each RNA contains open reading frames (ORFs) that are flanked by non-translated regions (NTRs). Figure 1 shows the genome organization and coding strategies of representative virus members from selected families in the Bunyavirales order.

For tri-segmented bunyaviruses, the L segment encodes L protein, the RNA-dependent RNA polymerase, and the M segment encodes the envelope glycoproteins $\mathrm{Gn}$ and $\mathrm{Gc}$, which are produced by the cleavage of the glycoprotein precursor. For some members of Peribunyaviridae, Nairoviridae, and Phenuiviridae, M segment encodes an additional nonstructural protein, NSm [7,11]. Processing of the glycoprotein precursor for CCHFV leads to the production of secreted non-structural glycoproteins [11]. The S segment encodes the nucleocapsid protein, N. For the families Peribunyaviridae and Phenuiviridae, the S segment also encodes the non-structural protein, NSs. The N and NSs proteins are encoded from two overlapping ORFs in Peribunyaviridae [7] and some members of the Hantaviridae [12]. For Phenuiviridae, N and NSs proteins are produced using an ambisense coding strategy [7]. The antigenomic S segment of most strains of CCHFV contains an additional ORF encoding NSs $[9,13,14]$.

The Arenaviridae family has a negative-sense, bi-segmented genome consisting of $\mathrm{L}$ and $\mathrm{S}$ segments, with the exception of the genus Antennavirus that contain a tri-segmented genome $[8,15]$. Each segment consists of two ORFs flanked by NTRs and separated by an intergenic region (IGR), utilizing an ambisense coding strategy. The $\mathrm{L}$ segment encodes $\mathrm{L}$ protein and the matrix protein, Z. Notably, arenaviruses within the genera Antennavirus and Hartmanivirus do not encode the $\mathrm{Z}$ protein $[15,16]$. The $\mathrm{S}$ segment encodes the nucleoprotein, NP, and the glycoprotein precursor complex, GPC, which is further cleaved to produce two glycoproteins [8].

\section{Bunyavirus reverse genetics systems}

Two different systems have been used to rescue infectious bunyaviruses from cloned cDNAs: RNA polymerase I/II system and the bacteriophage T7 RNA polymerase system. Rescue systems have been established for several viruses of the Arenaviridae, Nairoviridae, Peribunyaviridae, and Phenuiviridae families, but not for those in the Hantaviridae family (Figure 2). A minigenome rescue system for ANDV has been reported, however, the system encountered problems with reproducibility, possibly due to the unreliable expression levels of $\mathrm{L}$ protein as well as difficulties in achieving the expression of an optimal biological ratio of viral proteins [17]. In addition, there could be some unidentified viral or host-specific factor(s) that may be required for the establishment of a successful reverse genetics system for hantaviruses [17].

The successful rescue of a bunyavirus, entirely from plasmid DNA, using the RNA pol I/II system was first demonstrated for lymphocytic choriomeningitis virus (LCMV), a member of the Arenaviridae family [18-20]. This system utilizes the endogenous cellular RNA pol I 
to drive the synthesis of viral RNAs from cloned cDNAs in the nucleus. A careful consideration must be given to the selection of RNA pol I promoter and the cell line used for virus rescue using this system, as the RNA pol I promoter is species-specific. A potential drawback of this system for the rescue of cytoplasmic viruses is the possibility of splicing of pol II-driven mRNAs encoding viral proteins, which could affect the expression of viral proteins and the efficiency of virus rescue. Nonetheless, this system has been widely used for the successful rescue of infectious viruses in the Arenaviridae family, including LASV [21], MACV [22], and JUNV [20]. This system was also used for the live-attenuated MP-12 strain and wild-type ZH548 strain of RVFV [23]. Akabane virus (AKAV), in the

Peribunyaviridae family, was successfully rescued using this system [24], but was improved by utilizing the T7 pol system [25]. A higher transfection efficiency of the three plasmidbased T7 pol system compared to a five plasmid-based RNA Pol I-based system could have contributed to the improved rescue efficiency [25]. However, a study comparing both these reverse genetics systems for RVFV yielded similar rescue efficiencies [26].

The feasibility of the $\mathrm{T} 7$ pol system for the rescue of an infectious bunyavirus was demonstrated for the first time in Bunyamwera (BUNV) within the Peribunyaviridae family [27]. The T7 pol system, in which expressed $\mathrm{T} 7$ polymerase drives the transcription of viral RNAs from transfected plasmids in the cytoplasm, has been used to successfully rescue infectious viruses for several members in the bunyavirus order (Figure 2). These include the Arenaviridae family members, LASV [20,21], Pichinde virus (PICV) [20,28], LCMV, JUNV, and Lujo virus (LUJV) [20]; Peribunyaviridae family members, LACV [29], OROV [30], SBV [31], Cache valley virus (CVV) and Kairi virus (KRIV) [32]; Phenuiviridae family members, RVFV (strains ZH501, ZH548, and MP-12) [4,23], Uukuniemi (UUKV) [33], Arumowot (AMTV) [34], Toscana (TOSV) [35], and SFTSV [36]; and Nairoviridae family members CCHFV [11] and Hazara virus (HAZV) [37]. The T7 pol system is strictly dependent on the exogenous expression of $\mathrm{T} 7$ pol or cell lines, which stably express high levels of T7 pol. Although this system allows the rescue of bunyaviruses using cell lines from different species, many of these cell lines are not approved for vaccine development by the FDA [20].

\section{Investigation of Bunyavirus virulence factors using reverse genetics}

Reverse genetics approaches have been vital for the elucidation of virulence factors in bunyaviruses, which are important for the rational design of live-attenuated vaccines. NSs has been identified as a major virulence factor, which employs several strategies to suppress host innate immune responses [38]. For example, RVFV NSs inhibits host transcription, suppresses the activation of the IFN- $\beta$ promoter, and facilitates viral translation by degradation of the protein kinase, PKR [23,38]. SFTSV, carrying mutations in NSs, is unable to target the TPL2 signaling pathway and induces IL-10 production, leading to the incapability to antagonize the innate immune response [39]. NSs proteins of CVV, KRIV, OROV and SBV have IFN antagonizing functions [30-32]. Many functions of the NSs protein for other bunyaviruses have been reviewed by others and summarized in Table 1 [38]. NSm also serves as a viral virulence factor for some bunyavirus members such as RVFV, in which NSm has an anti-apoptotic function (Table 1) [23]. NSm deletion mutants for BUNV and AKAV showed an attenuated phenotype in cell-culture and BUNV NSm was 
shown to be involved in the formation of tubular virus-factories around the Golgi-complex $[40,41]$.

Arenavirus NP inhibits the RIG-I/MDA5 pathway by degrading dsRNAs through its exoribonuclease domain. Replication of LASV and LCMV mutants, lacking the exoribonuclease domain, resulted in type I IFN expression [2,42] and PICV mutant carrying a mutation in the exoribonuclease domain showed reduced pathogenesis in guinea pigs $[2,42]$. Z protein inhibits the IFN pathway by directly interacting with RIG-I and this effect is only evident in pathogenic strains [2,42]. In addition, the GPC encoded by the $\mathrm{S}$ segment, has also been shown to affect arenavirus replication in animals (Table 1) [42].

\section{Immune correlates of protection against bunyaviruses}

The mechanisms of immune-mediated protection against bunyaviruses have not been fully defined. The control of RVFV and SFTSV infections are primarily mediated by the production of neutralizing antibodies [4,43-45]. Humoral and/or cell-mediated immune responses provide protection against arenaviruses [2]. The correlates of protection against CCHFV remain poorly defined, as the induction of neutralizing antibodies does not seem to correlate with vaccine efficacy, and studies have focused on examining the protective roles of non-neutralizing antibodies and cell-mediated immunity [3,46]. The induction of neutralizing antibodies confers protection against LACV and SBV, although there is evidence suggesting the role of cell-mediated immunity in providing protection against secondary exposure to SBV $[10,47]$.

\section{Bunyavirus reverse genetics approaches for live-attenuated vaccine candidates}

\section{Vaccine candidates carrying mutations or deletions in specific viral genes}

Single-point mutations or deletions introduced into specific viral genes have been used for production of live-attenuated vaccine candidates against many bunyaviruses. Overattenuation, genetic instability of the introduced mutations as well as the introduction of compensatory mutations during large scale production that could potentially cause a reversion to virulent viruses are possible concerns with this approach. As NSs and NSm serve as virulent factors, deletion of NSs and/or NSm have been introduced into the wildtype RVFV ZH501 strain for the generation of live-attenuated vaccines. These viruses, ZH501- $\Delta$ NSs and ZH501- $\Delta \mathrm{NSs} / \Delta \mathrm{NSm}$, were safe and induced sterilizing immunity in mice, adult sheep, marmoset non-human primates (NHP) and pregnant ewes, including protection of fetuses from abortions and malformations [4,48]. Insertion of a nonviral sequence to these vaccine candidates could also be useful for the differentiation between infected and vaccinated animals (DIVA). Two variants of the MP-12 strain of RVFV, one lacking the expression of both NSm and the $78 \mathrm{kDa}$ accessory protein, and the other lacking the expression of NSs protein, were highly efficacious for the protection of vaccinated ruminants and mice, respectively, from wild-type RVFV challenge. However, the variant lacking NSs was not as immunogenic as the NSm/78 kDa deletion mutant or parental MP-12 in ruminants and needs further evaluation of its safety profile in pregnant animals [49]. 
Another approach to further attenuate the MP-12 strain was to introduce 584 silent mutations in the viral genome; all mice immunized with this virus were protected from lethal RVFV challenge [50]. Safety of MP-12 and its recombinant viruses were reviewed by Ikegami [51]. Two live-attenuated SFTSV vaccine candidates were developed by introducing mutations into the NSs gene; one contained a large deletion of the NSs gene and the other had a specific amino acid substitution mutation. Both induced strong neutralizing antibodies after a single dose immunization in aged ferrets and offered $100 \%$ protection against a crossgenotype lethal challenge [44]. Two recombinant SBV viruses, one lacking NSs and the other lacking NSs and NSm, had an attenuated virulence and after immunization, elicited neutralizing antibodies in cattle, and protected them from wild-type virus challenge. However, serial passaging of the former in cell culture resulted in a reversion to a virulent phenotype [52].

\section{Codon-deoptimization of viral genes}

Codon-deoptimization is the replacement of commonly used codons with non-preferred codons in the viral ORFs to decrease gene expression levels and reduce the possibility of reversion to virulence. Some drawbacks of this approach include genetic instability in vivo and reduced levels of viral growth, due to decreased viral protein expression, that can consequently impact the immunogenicity of the vaccine. This method has been used for the attenuation of LCMV and LASV [53,54]. Partially codon-deoptimized NP of LCMV resulted in reduced levels of NP expression, and attenuation of virulence in mice. Mice immunized with a single dose of this virus were fully protected against lethal wild-type virus challenge [55]. LMCV with a full codon-deoptimized glycoprotein showed lower GP expression levels, yet immunized mice were effectively protected from lethal challenge of wild-type virus [53]. A LASV mutant expressing codon-deoptimized GPC was highly attenuated in guinea pigs and provided complete protection against lethal challenge of LASV after a single dose immunization. Neutralizing antibodies were not detected in immunized guinea pigs, suggesting the role of cell-mediated immunity in protection against LASV [54].

\section{Single-cycle replicable vaccine candidates}

Single-cycle replicable viruses, or virus replicon particles (VRPs), are safe vaccine candidates as they do not spread beyond the initially infected cells. Although this vastly improves the safety profile of such vaccine candidates, a drawback of this approach could be the feasibility of generating high titers of VRPs required for immunogenicity and protective efficacy. By using RVFV strain 35/74, RVFV replicon particles (RRP) consisting of L and S RNAs, the latter of which encodes $\mathrm{N}$ and green fluorescent protein (GFP), were generated [56]. Due to the lack of M segment, the RRPs underwent a single cycle replication. All mice immunized with a single dose of the RRPs survived lethal wild-type RVFV challenge. Furthermore, sheep vaccinated with the RRPs were protected against lethal challenge. The RRPs were subsequently modified by expressing Gn, instead of GFP, from the S RNA [57]. Mice immunized with the modified RRPs induced higher neutralizing antibodies than the original RRP. Lambs immunized with a single dose of the modified RRPs had no viral RNA present in any tissue after wild-type virus challenge [45,57]. RRPs of RVFV strain ZH501 carrying L and S RNA, the latter of which expressed N and GFP, have been generated. The 
RRP lacked neurovirulence in suckling mice. Mice immunized with a single dose of RRP were fully protected against wild-type RVFV challenge [45,58].

LASV VRP, lacking the GPC gene, did not cause clinical manifestations in immunized guinea pigs and the immunized animals were protected from fever, weight loss, and lethality induced by wild-type virus challenge [59]. A single high dose immunization of CCHFV VRPs, carrying only the $\mathrm{L}$ and $\mathrm{S}$ segments, in mice lacking the IFN receptor had no clinical manifestations, implying CCHFV VRPs may serve as a safe CCHFV vaccine platform [60].

RVFV MP-12-based single-cycle replicable virus (scMP-12), that carried all three viral RNAs, has been reported. scMP-12 encoded N and GFP in the S segment and had a specific mutation abolishing the membrane fusion activity in Gc; scMP-12 undergoes a single-cycle replication due to inability of Gc-induced membrane fusion [45]. scMP-12 lacked neurovirulence, elicited neutralizing antibodies and protected the immunized mice from wild-type RVFV challenge [45]. Subsequently, an scMP-12 variant expressing a mutated NSs, which cannot inhibit host transcription but can degrade PKR, was generated. The immunogenicity and protective efficacy of this scMP-12 variant was better than the original scMP-12 [61].

\section{Viral genome rearrangement}

Rearrangement of the viral genome is another approach to attenuate viruses without changing the viral protein sequence. It would prevent the generation of revertant viruses and genomic reassortment between the vaccine and its wild-type virus, improving vaccine safety. A potential limitation is that the genome rearrangement could lead to low levels of viral protein expression, potentially reducing the overall immunogenicity of the vaccine candidate. A recombinant four segmented RVFV was generated by splitting the M segment into two M-like RNAs, one encoding Gn and the other encoding Gc (RVFV-4s). Although RVFV-4s encodes NSs, it was avirulent in mice. RVFV-4s elicited high neutralizing antibodies in immunized pregnant ewes and did not cross the ovine placental barrier [62]. Subsequently, RVFV-4s lacking NSs was generated and after a single dose immunization, young lambs were protected against lethal wild-type virus challenge $[62,63]$

A similar approach was used for arenaviruses, where the $\mathrm{S}$ segment was split into two RNAs, each carrying NP or GPC, resulting in a tri-segmented arenavirus. Splitting of the S segment allows the expression of additional proteins of interest, as well as creates a selective pressure to package all three RNA segments. This approach was first utilized for LCMV and JUNV Candid \#1. The tri-segmented viruses were highly attenuated in mice and the immunized mice exhibited protection against lethal virus challenge [64]. A similar approach was also applied to MACV, where the $\mathrm{S}$ segment was split to have two S RNAs: one carrying MACV NP with Chapare virus GPC and the other carrying MACV GPC with Guanarito virus GPC. Due to the introduction of an amino-acid deletion in Chapare and Guanarito virus GPCs, virus particles produced from infected cells only carried the glycoproteins of MACV. Additional mutations were also introduced into the NP and L proteins of this virus. The modified virus was moderately genetically stable and after immunization, 50\% of guinea pigs survived after challenge with wild-type JUNV [65]. 
By replacing the IGR of L segment (L-IGR) with the IGR of S segment (S-IGR) in LCMV, Iwasaki et al. found that the IGRs of arenaviruses are important for virus virulence; the mutant virus replicated efficiently in cell cultures but was highly attenuated in vivo. Mice immunized with this mutant virus were protected from lethal LCMV challenge.

Furthermore, mutant LCMVs, one replacing the L-IGR with a nonviral S-IGR like sequence and the other replacing the L-IGR with the S-IGR of a different arenavirus had attenuated virulence and elicited protective immunity [66]. Swapping the arenavirus ORFs was also used for attenuation of viral virulence. LCMV, carrying swapped NP ORF and GPC ORF in S RNA, had highly attenuated virulence in mice and a single immunization of this virus protected all mice from lethal wild-type virus challenge [67].

\section{Vaccine candidates with chimeric viral genes}

Recombinant chimeric bunyaviruses carrying genes derived from two different related viruses have been used as live-attenuated vaccine candidates. These chimeras can also potentially elicit cross-protective immunity. The potential drawbacks could be the instability of the inserted gene within the viral backbone as well as the incompatibility between viral proteins derived from different viruses. This approach was used to attenuate the RVFV ZH501 strain, which carried a chimeric S segment that encoded the NSs of related viruses, including TOSV, Punta toro virus (PTV) or Sandfly fever Sicilian virus (SFSV), or expressed a dominant negative PKR. The chimeras encoding SFSV NSs and dominant negative PKR were attenuated in mice, whereas those encoding the NSs of TOSV and PTV retained their virulence [68]. A recombinant LACV vaccine candidate was generated by replacing the ORF of LACV M segment with the corresponding ORF of a related Jamestown canyon virus (JCV), and thereby expressing JCV glycoproteins. The JCV/LACV chimeric virus lacked neurovirulence in mice and was highly immunogenic. Using an NHP model, the JCV/LACV chimeric virus was immunogenic and produced high neutralizing antibodies against JCV, LACV, and a related virus Tahyna virus (TAHV). The immunized NHPs were fully protected against level challenge with LACV, JCV, and TAHV [47]. These data suggest the use of a chimeric virus as a potential panperibunyavirus vaccine candidate. A chimeric MACV expressing the GPC of JUNV Candid \# 1 virus was fully attenuated and more immunogenic than Candid \# 1 in mice, inducing neutralizing antibodies, and provided full protection against wild-type MACV challenge [69,70].

Vaccine candidates that have the genome of a non-pathogenic virus backbone and express specific genes of a related pathogenic virus have been produced. Mopeia virus (MOPV), a non-pathogenic arenavirus, was used as a platform to express the GPCs of the pathogenic LASV. To create a hyperattenuated MOPV backbone, additional mutations were introduced into the exoribonuclease domain of NP, abrogating the type I IFN antagonizing function. Immunization of NHPs with a single dose of this chimera elicited a robust humoral and Tcell response and provided complete protection from LASV challenge. This approach may serve as a new vaccine platform for multiple pathogenic arenaviruses [71]. 


\section{Conclusions and future perspectives}

The establishment of bunyavirus reverse genetics system has facilitated an insight into the pathogenesis of bunyavirus infection through the identification of virulence factors. It also has provided a platform for the generation of live-attenuated vaccine candidates by using several strategies, which are summarized in Table 2. New advances in reverse genetics technology are overcoming the concerns for vaccine candidates to revert to virulent viruses and reassortment between a vaccine candidate and wild-type virus and allowed for the generation of vaccine candidates with potential DIVA markers. Moreover, generating recombinant bunyaviruses expressing additional genes of interest have facilitated the implementation of novel pan-bunyavirus vaccines and can potentially be used in identifying anti-bunyavirus drugs.

\section{Acknowledgments}

The cited studies that were performed in the authors' laboratory were supported by grants, AI114657, AI114657-S1 and AI127984, from the National Institutes of Health. BT was supported by a fellowship, T32-AI007526, from the National Institutes of Health.

\section{References}

Papers of special interest, published within the period of review, have been highlighted as

* of special interest

** of outstanding interest

1. Abudurexiti A, Adkins S, Alioto D, Alkhovsky SV, Avsic-Zupanc T, Ballinger MJ, Bente DA, Beer M, Bergeron E, Blair CD, et al.: Taxonomy of the order Bunyavirales: update 2019. Arch Virol 2019, 164:1949-1965. [PubMed: 31065850]

2. Brisse ME, Ly H: Hemorrhagic Fever-Causing Arenaviruses: Lethal Pathogens and Potent Immune Suppressors. Front Immunol 2019, 10:372. [PubMed: 30918506]

3. Dowall SD, Carroll MW, Hewson R: Development of vaccines against Crimean-Congo haemorrhagic fever virus. Vaccine 2017, 35:6015-6023. [PubMed: 28687403]

4. Fawzy M, Helmy YA: The One Health Approach is Necessary for the Control of Rift Valley Fever Infections in Egypt: A Comprehensive Review. Viruses 2019, 11.

5. Liu R, Ma H, Shu J, Zhang Q, Han M, Liu Z, Jin X, Zhang F, Wu X: Vaccines and Therapeutics Against Hantaviruses. Front Microbiol 2019, 10:2989. [PubMed: 32082263]

6. Sakkas H, Bozidis P, Franks A, Papadopoulou C: Oropouche Fever: A Review. Viruses 2018, 10.

7. Sun Y, Li J, Gao GF, Tien P, Liu W: Bunyavirales ribonucleoproteins: the viral replication and transcription machinery. Crit Rev Microbiol 2018, 44:522-540. [PubMed: 29516765]

8. Hallam SJ, Koma T, Maruyama J, Paessler S: Review of Mammarenavirus Biology and Replication. Front Microbiol 2018, 9:1751. [PubMed: 30123198]

9. Zivcec M, Scholte FE, Spiropoulou CF, Spengler JR, Bergeron E: Molecular Insights into CrimeanCongo Hemorrhagic Fever Virus. Viruses 2016, 8:106. [PubMed: 27110812]

10. Endalew AD, Faburay B, Wilson WC, Richt JA: Schmallenberg Disease-A Newly Emerged Culicoides-borne Viral Disease of Ruminants. Viruses 2019, 11.

11. Bergeron E, Zivcec M, Chakrabarti AK, Nichol ST, Albarino CG, Spiropoulou CF: Recovery of Recombinant Crimean Congo Hemorrhagic Fever Virus Reveals a Function for Non-structural Glycoproteins Cleavage by Furin. PLoS Pathog 2015, 11:e1004879. [PubMed: 25933376]

12. Mittler E, Dieterle ME, Kleinfelter LM, Slough MM, Chandran K, Jangra RK: Hantavirus entry: Perspectives and recent advances. Adv Virus Res 2019, 104:185-224. [PubMed: 31439149] 
13. Hewson R, Chamberlain J, Mioulet V, Lloyd G, Jamil B, Hasan R, Gmyl A, Gmyl L, Smirnova SE, Lukashev A, et al.: Crimean-Congo haemorrhagic fever virus: sequence analysis of the small RNA segments from a collection of viruses world wide. Virus Res 2004, 102:185-189. [PubMed: 15084400]

14. Barnwal B, Karlberg H, Mirazimi A, Tan YJ: The Non-structural Protein of Crimean-Congo Hemorrhagic Fever Virus Disrupts the Mitochondrial Membrane Potential and Induces Apoptosis. J Biol Chem 2016, 291:582-592. [PubMed: 26574543]

15. Shi M, Lin XD, Chen X, Tian JH, Chen LJ, Li K, Wang W, Eden JS, Shen JJ, Liu L, et al.: Author Correction: The evolutionary history of vertebrate RNA viruses. Nature 2018, 561:E6. [PubMed: 29946168]

16. Garry CE, Garry RF: Proteomics Computational Analyses Suggest that the Antennavirus Glycoprotein Complex Includes a Class I Viral Fusion Protein (alpha-Penetrene) with an Internal Zinc-Binding Domain and a Stable Signal Peptide. Viruses 2019, 11.

17. Brown KS, Ebihara H, Feldmann H: Development of a minigenome system for Andes virus, a New World hantavirus. Arch Virol 2012, 157:2227-2233. [PubMed: 22821183]

18. Flatz L, Bergthaler A, de la Torre JC, Pinschewer DD: Recovery of an arenavirus entirely from RNA polymerase I/II-driven cDNA. Proc Natl Acad Sci U S A 2006, 103:4663-4668. [PubMed: 16537369]

19. Martinez-Sobrido L, Cheng BY, de la Torre JC: Reverse Genetics Approaches to Control Arenavirus. Methods Mol Biol 2016, 1403:313-351. [PubMed: 27076139]

20. Ortiz-Riano E, Cheng BYH, Carlos de la Torre J, Martinez-Sobrido L: Arenavirus reverse genetics for vaccine development. J Gen Virol 2013, 94:1175-1188. [PubMed: 23364194]

21. Martinez-Sobrido L, Paessler S, de la Torre JC: Lassa Virus Reverse Genetics. Methods Mol Biol 2017, 1602:185-204. [PubMed: 28508222]

22. Patterson M, Seregin A, Huang C, Kolokoltsova O, Smith J, Miller M, Smith J, Yun N, Poussard A, Grant A, et al.: Rescue of a recombinant Machupo virus from cloned cDNAs and in vivo characterization in interferon (alphabeta/gamma) receptor double knockout mice. J Virol 2014, 88:1914-1923. [PubMed: 24284323]

23. Bouloy M, Flick R: Reverse genetics technology for Rift Valley fever virus: current and future applications for the development of therapeutics and vaccines. Antiviral Res 2009, 84:101-118. [PubMed: 19682499]

24. Ogawa Y, Sugiura K, Kato K, Tohya Y, Akashi H: Rescue of Akabane virus (family Bunyaviridae) entirely from cloned cDNAs by using RNA polymerase I. J Gen Virol 2007, 88:3385-3390. [PubMed: 18024908]

25. Takenaka-Uema A, Sugiura K, Bangphoomi N, Shioda C, Uchida K, Kato K, Haga T, Murakami S, Akashi H, Horimoto T: Development of an improved reverse genetics system for Akabane bunyavirus. J Virol Methods 2016, 232:16-20. [PubMed: 26927704]

26. Habjan M, Penski N, Spiegel M, Weber F: T7 RNA polymerase-dependent and - independent systems for cDNA-based rescue of Rift Valley fever virus. J Gen Virol 2008, 89:2157-2166. [PubMed: 18753225]

27. Bridgen A, Elliott RM: Rescue of a segmented negative-strand RNA virus entirely from cloned complementary DNAs. Proc Natl Acad Sci U S A 1996, 93:15400-15404. [PubMed: 8986823]

28. Dhanwani R, Huang Q, Lan S, Zhou Y, Shao J, Liang Y, Ly H: Establishment of Bisegmented and Trisegmented Reverse Genetics Systems to Generate Recombinant Pichinde Viruses. Methods Mol Biol 2018, 1604:247-253. [PubMed: 28986840]

29. Blakqori G, Weber F: Efficient cDNA-based rescue of La Crosse bunyaviruses expressing or lacking the nonstructural protein NSs. J Virol 2005, 79:10420-10428. [PubMed: 16051834]

30. Tilston-Lunel NL, Acrani GO, Randall RE, Elliott RM: Generation of Recombinant Oropouche Viruses Lacking the Nonstructural Protein NSm or NSs. J Virol 2015, 90:2616-2627. [PubMed: 26699638]

31. Elliott RM, Blakqori G, van Knippenberg IC, Koudriakova E, Li P, McLees A, Shi X, Szemiel AM: Establishment of a reverse genetics system for Schmallenberg virus, a newly emerged orthobunyavirus in Europe. J Gen Virol 2013, 94:851-859. [PubMed: 23255627] 
32. Dunlop JI, Szemiel AM, Navarro A, Wilkie GS, Tong L, Modha S, Mair D, Sreenu VB, Da Silva Filipe A, Li P, et al.: Development of reverse genetics systems and investigation of host response antagonism and reassortment potential for Cache Valley and Kairi viruses, two emerging orthobunyaviruses of the Americas. PLoS Neg1 Trop Dis 2018, 12:e0006884. [PubMed: 30372452]

33. Rezelj VV, Overby AK, Elliott RM: Generation of mutant Uukuniemi viruses lacking the nonstructural protein NSs by reverse genetics indicates that NSs is a weak interferon antagonist. $\mathrm{J}$ Virol 2015, 89:4849-4856. [PubMed: 25673721]

34. Hallam HJ, Lokugamage N, Ikegami T: Rescue of infectious Arumowot virus from cloned cDNA: Posttranslational degradation of Arumowot virus NSs protein in human cells. PLoS Negl Trop Dis 2019, 13:e0007904. [PubMed: 31751340]

35. Alexander AJT, Confort MP, Desloire S, Dunlop JI, Kuchi S, Sreenu VB, Mair D, Wilkie GS, Da Silva Filipe A, Brennan B, et al.: Development of a Reverse Genetics System for Toscana Virus (Lineage A). Viruses 2020, 12.

36. Brennan B, Li P, Zhang S, Li A, Liang M, Li D, Elliott RM: Reverse genetics system for severe fever with thrombocytopenia syndrome virus. J Virol 2015, 89:3026-3037. [PubMed: 25552716]

37. Fuller J, Surtees RA, Slack GS, Mankouri J, Hewson R, Barr JN: Rescue of Infectious Recombinant Hazara Nairovirus from cDNA Reveals the Nucleocapsid Protein DQVD Caspase Cleavage Motif Performs an Essential Role other than Cleavage. J Virol 2019, 93.

38. Ly HJ, Ikegami T: Rift Valley fever virus NSs protein functions and the similarity to other bunyavirus NSs proteins. Virol J 2016, 13:118. [PubMed: 27368371]

39. Choi Y, Park SJ, Sun Y, Yoo JS, Pudupakam RS, Foo SS, Shin WJ, Chen SB, Tsichlis PN, Lee WJ, et al.: Severe fever with thrombocytopenia syndrome phlebovirus non-structural protein activates TPL2 signalling pathway for viral immunopathogenesis. Nat Microbiol 2019, 4:429-437. [PubMed: 30617349]

40. Ishihara Y, Shioda C, Bangphoomi N, Sugiura K, Saeki K, Tsuda S, Iwanaga T, Takenaka-Uema A, Kato K, Murakami S, et al.: Akabane virus nonstructural protein NSm regulates viral growth and pathogenicity in a mouse model. J Vet Med Sci 2016, 78:1391-1397. [PubMed: 27181086]

41. Kraatz F, Wernike K, Reiche S, Aebischer A, Reimann I, Beer M: Schmallenberg virus nonstructural protein NSm: Intracellular distribution and role of non-hydrophobic domains. Virology 2018, 516:46-54. [PubMed: 29329078]

42. Shao J, Liang Y, Ly H: Human hemorrhagic Fever causing arenaviruses: molecular mechanisms contributing to virus virulence and disease pathogenesis. Pathogens 2015, 4:283-306. [PubMed: 26011826]

43. Faburay B, LaBeaud AD, McVey DS, Wilson WC, Richt JA: Current Status of Rift Valley Fever Vaccine Development. Vaccines (Basel) 2017, 5.

44. Yu KM, Park SJ, Yu MA, Kim YI, Choi Y, Jung JU, Brennan B, Choi YK: Cross-genotype protection of live-attenuated vaccine candidate for severe fever with thrombocytopenia syndrome virus in a ferret model. Proc Natl Acad Sci U S A 2019.* The authors assess the efficacy of two live-attenuated SFTSV vaccine candidates in an immunocompetent aged-ferret model that recapitulates the pathology of SFTS disease. Both viruses were able to induce a humoral immune response and confer full protection against a cross-genotype lethal challenge.

45. Terasaki K, Tercero BR, Makino S: Single-cycle replicable Rift Valley fever virus mutants as safe vaccine candidates. Virus Res 2016, 216:55-65. [PubMed: 26022573]

46. Goedhals D, Paweska JT, Burt FJ: Long-lived CD8+ T cell responses following Crimean-Congo haemorrhagic fever virus infection. PLoS Negl Trop Dis 2017, 11:e0006149. [PubMed: 29261651]

47. Bennett RS, Gresko AK, Nelson JT, Murphy BR, Whitehead SS: A recombinant chimeric La Crosse virus expressing the surface glycoproteins of Jamestown Canyon virus is immunogenic and protective against challenge with either parental virus in mice or monkeys. J Virol 2012, 86:420 426. [PubMed: 22013033]

48. Smith DR, Johnston SC, Piper A, Botto M, Donnelly G, Shamblin J, Albarino CG, Hensley LE, Schmaljohn C, Nichol ST, et al.: Attenuation and efficacy of live-attenuated Rift Valley fever virus vaccine candidates in non-human primates. PLoS Negl Trop Dis 2018, 12:e006474. [PubMed: $29742102] * *$ The authors evaluated the efficacy of two RVFV live-attenuated vaccine candidates 
that contain complete deletions of two known RVFV virulence factors. The vaccines were safe and effective in NHPs, highlighting the potential for further development of these candidates for human use.

49. Morrill JC, Laughlin RC, Lokugamage N, Pugh R, Sbrana E, Weise WJ, Adams LG, Makino S, Peters CJ: Safety and immunogenicity of recombinant Rift Valley fever MP-12 vaccine candidates in sheep. Vaccine 2013, 31:559-565. [PubMed: 23153443]

50. Ly HJ, Nishiyama S, Lokugamage N, Smith JK, Zhang L, Perez D, Juelich TL, Freiberg AN, Ikegami T: Attenuation and protective efficacy of Rift Valley fever phlebovirus rMP12-GM50 strain. Vaccine 2017, 35:6634-6642. [PubMed: 29061350]

51. Ikegami T: Rift Valley fever vaccines: an overview of the safety and efficacy of the live-attenuated MP-12 vaccine candidate. Expert Rev Vaccines 2017, 16:601-611. [PubMed: 28425834]

52. Kraatz F, Wernike K, Hechinger S, Konig P, Granzow H, Reimann I, Beer M: Deletion mutants of Schmallenberg virus are avirulent and protect from virus challenge. J Virol 2015, 89:1825-1837. [PubMed: 25410877]

53. Cheng BYH, Nogales A, de la Torre JC, Martinez-Sobrido L: Development of live-attenuated arenavirus vaccines based on codon deoptimization of the viral glycoprotein. Virology 2017, 501:35-46. [PubMed: 27855284]

54. Cai Y, Ye C, Cheng B, Nogales A, Iwasaki M, Yu S, Cooper K, Liu DX, Hart R, Adams R, et al.: A Lassa Fever Live-Attenuated Vaccine Based on Codon Deoptimization of the Viral Glycoprotein Gene. mBio 2020, 11.** The authors report the generation of a novel codon-deoptimized liveattenuated LASV vaccine candidate that was immunogenic and safe in a guinea pig model, which supports further testing in a NHP model.

55. Cheng BY, Ortiz-Riano E, Nogales A, de la Torre JC, Martinez-Sobrido L: Development of liveattenuated arenavirus vaccines based on codon deoptimization. J Virol 2015, 89:3523-3533. [PubMed: 25589652]

56. Kortekaas J, Antonis AF, Kant J, Vloet RP, Vogel A, Oreshkova N, de Boer SM, Bosch BJ, Moormann RJ: Efficacy of three candidate Rift Valley fever vaccines in sheep. Vaccine 2012, 30:3423-3429. [PubMed: 22449427]

57. Oreshkova N, van Keulen L, Kant J, Moormann RJ, Kortekaas J: A single vaccination with an improved nonspreading Rift Valley fever virus vaccine provides sterile immunity in lambs. PLoS One 2013, 8:e77461. [PubMed: 24167574]

58. Dodd KA, Bird BH, Metcalfe MG, Nichol ST, Albarino CG: Single-dose immunization with virus replicon particles confers rapid robust protection against Rift Valley fever virus challenge. J Virol 2012, 86:4204-4212. [PubMed: 22345465]

59. Kainulainen MH, Spengler JR, Welch SR, Coleman-McCray JD, Harmon JR, Klena JD, Nichol ST, Albarino CG, Spiropoulou CF: Use of a Scalable Replicon-Particle Vaccine to Protect Against Lethal Lassa Virus Infection in the Guinea Pig Model. J Infect Dis 2018, 217:1957-1966. [PubMed: 29800368]

60. Scholte FEM, Spengler JR, Welch SR, Harmon JR, Coleman-McCray JD, Freitas BT, Kainulainen MH, Pegan SD, Nichol ST, Bergeron E, et al.: Single-dose replicon particle vaccine provides complete protection against Crimean-Congo hemorrhagic fever virus in mice. Emerg Microbes Infect 2019, 8:575-578. [PubMed: 30947619] * The authors report the first production of a CCHF virus-like replicon particle vaccine candidate using an established reverse genetics system that can be used as a vaccine platform against CCHFV.

61. Terasaki K, Juelich TL, Smith JK, Kalveram B, Perez DD, Freiberg AN, Makino S: A single-cycle replicable Rift Valley fever phlebovirus vaccine carrying a mutated NSs confers full protection from lethal challenge in mice. Sci Rep 2018, 8:17097. [PubMed: 30459418]

62. Wichgers Schreur PJ, van Keulen L, Kant J, Kortekaas J: Four-segmented Rift Valley fever virusbased vaccines can be applied safely in ewes during pregnancy. Vaccine 2017, 35:3123-3128. [PubMed: 28457675]

63. Wichgers Schreur PJ, Kant J, van Keulen L, Moormann RJ, Kortekaas J: Four-segmented Rift Valley fever virus induces sterile immunity in sheep after a single vaccination. Vaccine 2015, 33:1459-1464. [PubMed: 25665959] 
64. Emonet SF, Seregin AV, Yun NE, Poussard AL, Walker AG, de la Torre JC, Paessler S: Rescue from cloned cDNAs and in vivo characterization of recombinant pathogenic Romero and liveattenuated Candid \#1 strains of Junin virus, the causative agent of Argentine hemorrhagic fever disease. J Virol 2011, 85:1473-1483. [PubMed: 21123388]

65. Zaza AD, Herbreteau CH, Peyrefitte CN: Description and characterization of a novel liveattenuated tri-segmented Machupo virus in Guinea pigs. Virol J 2018, 15:99.

66. Iwasaki M, Cubitt B, Sullivan BM, de la Torre JC: The High Degree of Sequence Plasticity of the Arenavirus Noncoding Intergenic Region (IGR) Enables the Use of a Nonviral Universal Synthetic IGR To Attenuate Arenaviruses. J Virol 2016, 90:3187-3197. [PubMed: 26739049]

67. Cheng BY, Ortiz-Riano E, de la Torre JC, Martinez-Sobrido L: Arenavirus Genome Rearrangement for the Development of Live Attenuated Vaccines. J Virol 2015, 89:7373-7384. [PubMed: 25972555]

68. Nishiyama S, Slack OA, Lokugamage N, Hill TE, Juelich TL, Zhang L, Smith JK, Perez D, Gong B, Freiberg AN, et al.: Attenuation of pathogenic Rift Valley fever virus strain through the chimeric S-segment encoding sandfly fever phlebovirus NSs or a dominant-negative PKR. Virulence 2016, 7:871-881. [PubMed: 27248570]

69. Koma T, Patterson M, Huang C, Seregin AV, Maharaj PD, Miller M, Smith JN, Walker AG, Hallam S, Paessler S: Machupo Virus Expressing GPC of the Candid\#1 Vaccine Strain of Junin Virus Is Highly Attenuated and Immunogenic. J Virol 2016, 90:1290-1297. [PubMed: 26581982]

70. Koma T, Huang C, Aronson JF, Walker AG, Miller M, Smith JN, Patterson M, Paessler S: The Ectodomain of Glycoprotein from the Candid\#1 Vaccine Strain of Junin Virus Rendered Machupo Virus Partially Attenuated in Mice Lacking IFN-alphabeta/gamma Receptor. PLoS Negl Trop Dis 2016, 10:e0004969. [PubMed: 27580122]

71. Carnec X, Mateo M, Page A, Reynard S, Hortion J, Picard C, Yekwa E, Barrot L, Barron S, Vallve A, et al.: A Vaccine Platform against Arenaviruses Based on a Recombinant Hyperattenuated Mopeia Virus Expressing Heterologous Glycoproteins. J Virol 2018, 92.** The authors report the generation of a promising vaccine platform for pathogenic arenaviruses based on a hyperattenuated strain of a non-pathogenic arenavirus backbone, which was safe and immunogenic in NHPs. 


\section{Highlights}

- Summary of the currently established reverse genetics approaches for bunyaviruses

- Review of bunyavirus virulence factors determined by reverse genetics

- Evaluation of novel live-attenuated vaccine candidates against bunyaviruses 


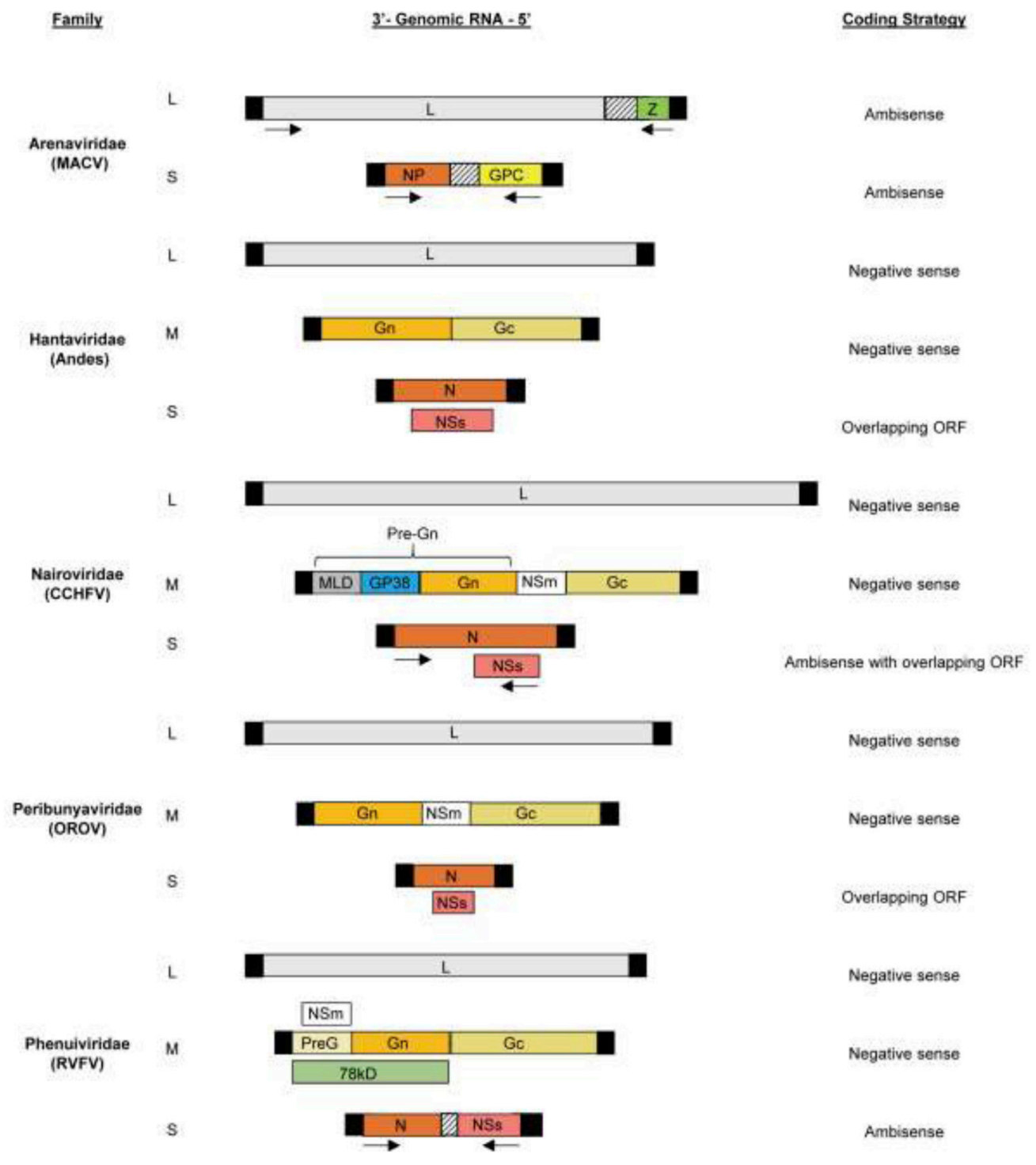

Figure 1.

Schematic diagram of the genome organization and coding strategies of representative virus members from selected families in the Bunyavirales order. The viral proteins encoded by each of the viral RNA segments are indicated within the boxes. The black boxes represent the terminal NTRs for each viral RNA segment. The intergenic regions are indicated by boxes with dashed lines. The figure is not drawn to scale. MLD; mucin-like domain. 


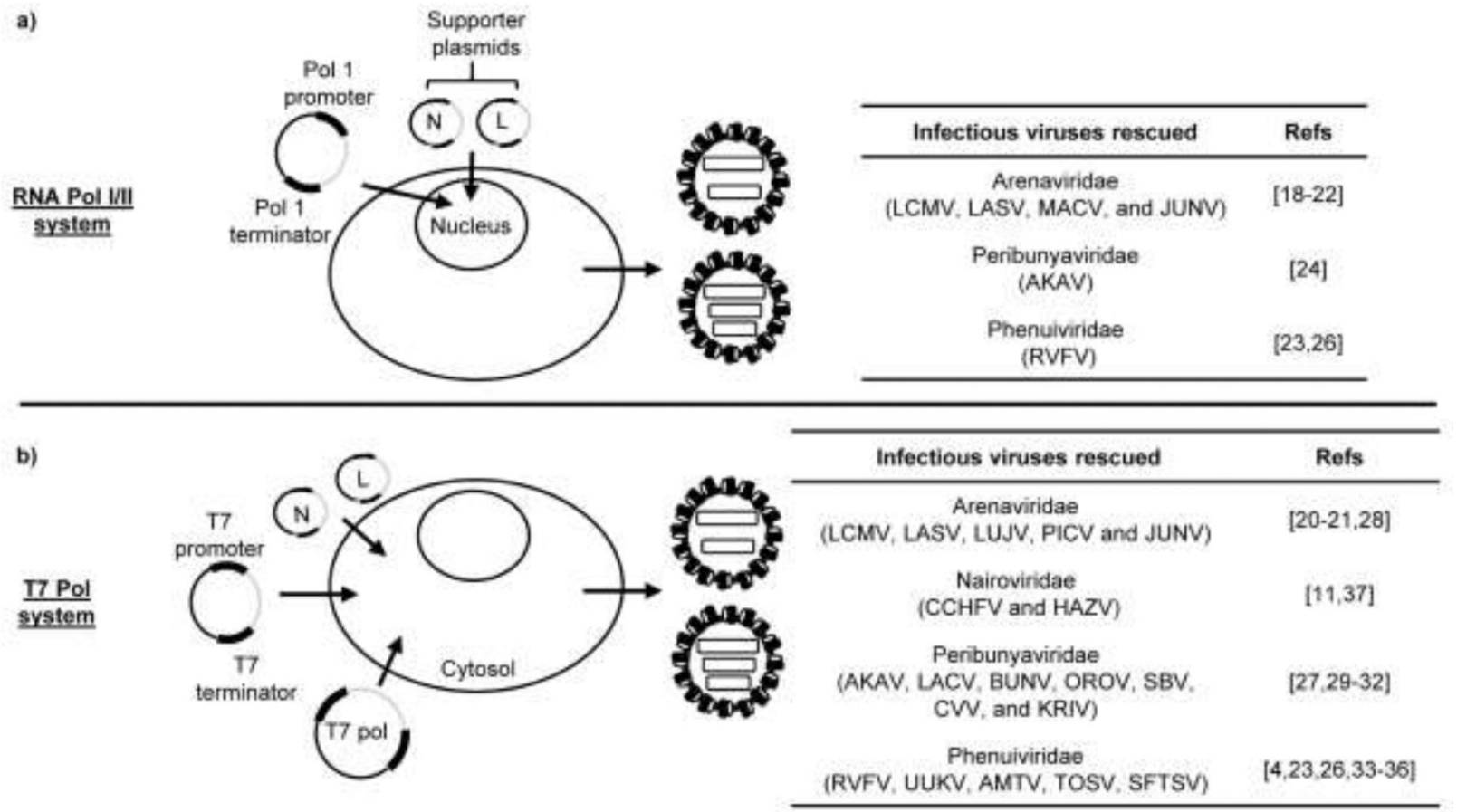

Figure 2.

Overview of the reverse genetics systems for the generation of infectious bunyaviruses. (a) Schematic representation of the RNA polymerase (Pol) I/II-driven reverse genetics system. Plasmids, encoding the viral RNA segments under the control of cellular RNA pol I promoter, are transfected along with RNA pol II-driven supporter plasmids expressing the nucleocapsid protein, $\mathrm{N}$, and viral RNA polymerase, L, into permissive mammalian cells. The viral RNA segments, which are synthesized from the RNA pol I-driven plasmids in the nucleus, undergo replication and transcription, mediated by the expressed $\mathrm{N}$ and $\mathrm{L}$ proteins, resulting in the amplification of viral RNA genome and production of viral proteins required for the successful recovery of infectious viruses. The right panel indicates the infectious bunyaviruses rescued using the RNA Pol I/II system along with the corresponding references (Refs). (b) Schematic representation of the T7 pol-driven reverse genetics system. Plasmids, encoding the viral RNA segments under the control of T7 pol-driven promoter, are cotransfected with supporter plasmids expressing $\mathrm{N}$ and L proteins into suitable cells transiently or stably expressing T7 RNA polymerase. The expressed N and L proteins drive the replication and transcription of viral RNA segments, which are produced from the T7 pol-driven plasmids in the cytoplasm, resulting in the accumulation of viral RNA genome and viral proteins necessary for the recovery of infectious viruses. The panel on the right displays the infectious bunyaviruses rescued using the T7 Pol system along with the corresponding references. 
Table 1:

Bunyavirus virulence factors as determined by reverse genetics

\begin{tabular}{llll}
\hline Family & Virulence factors & Function $(\mathbf{s})$ & Refs \\
\hline Arenaviridae & NP & IFN antagonist & {$[2,42]$} \\
& Z & IFN antagonist & {$[2,42]$} \\
& GPC & Virus replication & {$[42]$} \\
Peribunyaviridae & NSs & IFN antagonist & {$[30-32]$} \\
Phenuiviridae & NSs & Inhibition of host transcription, PKR degradation, and IFN antagonist & {$[23,35,38-39]$} \\
& NSm & Anti-apoptotic & {$[23]$} \\
\hline
\end{tabular}


Table 2:

Bunyavirus reverse genetics approaches for live-attenuated vaccines

\begin{tabular}{llll}
\hline Approach & Virus & Tested animal model & Refs \\
\hline Mutation/Deletions & SBV & Cattle & {$[52]$} \\
& RVFV & Mice, sheep, pregnant ewes, marmoset non-human primate (NHP) & {$[4,48-51]$} \\
& SFTSV & Aged-ferret & {$[44]$} \\
Codon-deoptimization & LCMV & Mice & {$[53,55]$} \\
& LASV & Guinea pig & {$[54]$} \\
Single-cycle replicable virus & LASV & Guinea pig & {$[59]$} \\
& CCHFV & Type I IFN receptor knockout mice & {$[60]$} \\
& RVFV & Mice, sheep, lambs, suckling mice & {$[45,56-58,61]$} \\
Viral genome rearrangement & LCMV & Mice & {$[64,66-67]$} \\
& MACV & Guinea pig & {$[65]$} \\
& JUNV & Mice & {$[64]$} \\
& RVFV & Mice, lambs, pregnant ewes & {$[62-63]$} \\
& LASV & NHP & {$[71]$} \\
& MACV & Mice & {$[69-70]$} \\
& LACV & Mice, NHP & {$[47]$} \\
& RVFV & Mice & {$[68]$} \\
\hline
\end{tabular}

